\title{
Design and Development of Impeller Synergic Systems of Electromagnetic Type to Levitation/Suspension Flight of Symmetrical Bodies
}

\author{
Francisco Bulnes ${ }^{1}$, Juan Carlos Maya ${ }^{2}$, Isaías Martínez ${ }^{3}$ \\ ${ }^{1}$ Department of Research in Mathematics and Engineering, TESCHA, Chalco, Mexico; ${ }^{2}$ Division of Computational Systems Engi- \\ neering, TESCHA, Chalco, Mexico; ${ }^{3}$ Division of Electronic and Communications Engineering, TESCHA, Chalco, Mexico. \\ Email: francisco.bulnes@tesch.edu.mx, mayacastell@yahoo.com.mx
}

Received November $4^{\text {th }}, 2011$; revised December $6^{\text {th }}, 2011$; accepted December $26^{\text {th }}, 2011$

\begin{abstract}
Using certain models of twistor surfaces for fields of force and the mathematical relationships that lie among fields, lines, surfaces and flows of energy, it has been designed and developed a flight electromagnetic type system based on the synergic study of their electromagnetic field geodesics to generate vehicle levitation, suspension and movement without being in contact with the surface. The idea of such work is to obtain a new flight and impulse patent of an electromagnetic vehicle by principles of super-conduction and some laws of the current like Eddy currents and principles which are very similar to mechanics of sidereal objects like galaxies or stars under models of twistor surfaces. This vehicle will be controlled by one microchip that will be programmed by conscience operators algebra of electromagnetic type that leads to the flow of Eddy currents, the iso-rotations and suspension of the special geometrical characteristics vehicle, generating also on the vehicle structure certain "magnetic conscience" that provokes all movements like succeeding to the sidereal objects in the universe.
\end{abstract}

Keywords: Electromagnetic Levitation; Twistor Surfaces of Impulse; Symmetrical Space of Iso-Rotations; Superconductive Quantum Algebra; Magnetic Conscience Operators

\section{Introduction}

The first consideration towards constructing a field propulsion system would likely be allowed for it to have a very strong field [1-4]. Now most electromagnetic fields radiate so that a strong field can be achieved, a continuous amount of energy it also must be fed in the form of power from a reliable and autonomous source. Thus it is necessary to realize an appropriate research on superconducting materials that joint with a magnetic field created in the reactor, which produces a permanent diamagnetic effect and whose repulsion force of the magnetic field with the superconductor be expelled and controlled for a rotation ring whose movement must generate and feed the electromagnetic remnants of this same interaction inducing the momentum of impulse through a momentum of torsion produced by the electromagnetic forces [5-8]. This imitating the forces that conform and generate the sidereal objects movements. This type of engendered by magnetic fields and superconductor materials in the universe create all the movements in sidereal objects like stars and galaxies following an intrinsic geometry due to these fields [6,9], for example, the ga- laxy is moved on a magnetic disk with their spiral arms alignment due to their intersidereal magnetic field, their rotation that is engendered for magnetic field and their interaction with the superconductor materials, creating an electromagnetic rotation as described by the Helmholtz theorem $[1,6,10]$, or their evolution from their formation passing for several forms from the ellipsoid in their early stage until their adult stage with form of spiral disk or plate $[9,10]$, see the Figure 1. A topological study made before [8], demonstrates the existent isomorphism between three fundamental geometrical forms that are propitious for their intrinsic relationship with the studies on superconductors $[11,12]$, and the natural evolution of these geometrical forms with electromagnetic energy born from magnetic fields and superconductors studies in magneto-hydrodynamics of the space $[5,6,8,10]$, which are a sphere, an ellipsoid and a disk, where applying the formula of the magnetic momentum $[8,10]$ :

$$
M=V M=1 / 3 a b c\left(\mu^{(\mathrm{i})}-1\right) H_{z} /\left[1+\left(\mu^{(\mathrm{i})}-1\right) n^{(z)}\right]
$$

and using the magnetic energy of the superconductor in an external magnetic field perpendicular at a plate (disc): 


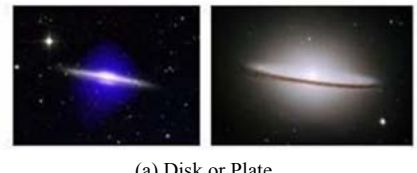

(a) Disk or Plate

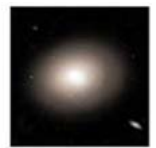

(b) Elipsoidal

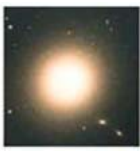

(c) Spherical
Figure 1. Three geometrical forms in the sidereal space corresponding to the three forms demonstrated topologically in the lemma by $F$. Bulnes (orbital spaces) [2,9,10,13], and lemma by L. Landau (magnetic momentum) [14]. Observe the natural accretion rings that conform the galaxy in a stage of development of $[10,15]$.

$$
E_{M}=-1 / 2 H M=r^{3} H^{2} / 3 \pi,[17],
$$

Then the space engendered by the perpendicular field of the disk results in isomorphic (in the context of electromagnetic actions), to the space of field $H_{c}(1-n)$, which is an ellipsoid $[8,10]$. If in ellipsoid $a=b=c$, then we have a sphere of the electromagnetic nature. Now it is just necessary to consider the electromagnetic performance to navigation and horizontal displacement of ship without effects irradiative due to the strong electromagnetic forces [4-6].

A good intent to build a strong local non-radiating electromagnetic field is to build it as a standing wave in the flight of horizontal displacement with a permanent effect of magnetic levitation and suspension in the space, but outside enclave to part of any field that exists in the media $[1,4]$. The electromagnetic part standing wave field does not radiate. In this point we've got in past researches that the electromagnetic field of impulse and levitation that comes of the interaction superconductormagnetic field must place a shield in the structure of the vehicle that to nano-metric level produces the effects of levitation and suspension required to their flight $[8,12]$, but with the iso-rotations established to the movement like a wave through altering the actions of the fields in their interaction reactor-rotor/ring of the vehicle [8]. The orbital spaces due to the ring rotation of the vehicle, as shown in $[8,11]$, magnetic torus must be to conform the twistor surface to govern the movement type wave described before. To produce the actions like a traveler wave a control would be designed with micro-controller that repeat until the voltage/current peaks and nodes achieve the amplitude wanted that comes of Eddy currents using the sustentation of the vehicle $[8,10]$. If the shorted-end by the Eddy current to a version of transmission line to produce the displacements using to produce standing magneto-hydrodynamics waves $[6,8]$, the current would be maximum and the voltage minimum at the shorter end of the line. Now well, if we rotate this shorted end about the location of the input of the line such as the short travels 360 degrees around the input to the transmission line, (as described by the orbital spaces gene- rated by rotation $\mathrm{C}(\mathbb{C})$, of our vehicle), the result is a plate shape, see Figure 2. This structure goes with the designs of our prototypes see Figure 3, under laws of lemma by $F$. Bulnes [8,10]. Therefore, the $\mathbb{E} \otimes \mathfrak{I}$-field is the defining shape for a plate even if there is no body apparently. In this last point, and using the fact of the vehicle due turn in a perfect diamagnetic object $[5,8,12]$, with a superconducting penetration of the type II, $[6,8]$, it is necessary the implementation of an alloy whose material of the reactor be a superconductor to conditions of ambient temperature. But that only will obtain developing in each particle of the structure of the vehicle a "magnetic conscience" defined into the programming of the vehicle that establishes a microscopic magnetic flux for particle of the vehicle $[8,12]$. In few words: to create the shape of spiral form on vehicle does not exist, and vice versa, whose shape will be integrated (by our synergic electro-dynamical principles), like the twistor surface of movement and total flight $[2,4,8]$.

\section{Synergic Electrodynamical Principles}

If we consider that the Maxwell equations can also be obtained like solution to a variational problem, then due to the actions of the fields $F$, that produce the movement of all the particles in the space $M$, and using the corresponding vector bundle of these electromagnetic field to define it gemetrically, we can define those action as [1, 16]:

$$
\mathfrak{I}: T M \rightarrow R,
$$

with rule of correspondence

$$
\mathfrak{I}(x(s)):=\text { FluxL }_{\text {MAX }}(x(s)) x(s),
$$

and the energy due to $L_{M A X}$, is

$$
E=\mathfrak{I}-L_{\text {MAX }},
$$

If we consider the global evaluation of the operator that acts on the space of points $x(s)$, this comes like

$$
\mathfrak{J}:(x(s)) \mapsto \int_{M} L_{M A X}(x(s)) \mathrm{d}(x(s)) .
$$

The integral Equation (6), represents the action $\mathfrak{I}(\gamma)$, by the Hamilton principle that establishes that the particle will cover that trajectory that minimizes the action. Likewise, the equation of movement comes pre-determined for the minimum of this action $\mathfrak{I}(\gamma)$, and pre-established for the variation condition [17]:

$$
\delta\left(\int_{\gamma} L_{M A X}(x(s), \dot{x}(s)) \mathrm{d} s\right)=\int_{x\left(s_{0}\right)}^{x\left(s_{1}\right)} \delta\left(\mathfrak{I}_{E C}+\mathfrak{I}_{E P i} \delta q^{i}\right) \mathrm{d} s .(7)
$$

In particular, if we want the evaluation of this action of Maxwell along an elected trajectory into the field of minimal trajectories, by the principle of the minimal action, 


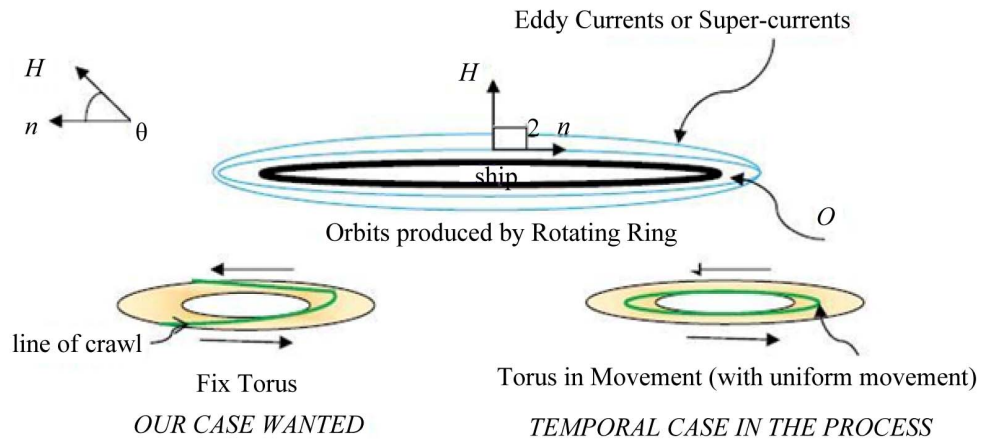

Figure 2. Orbits required to conform the twistor surface to magnetic impulse of the ship proposed. This orbits belongs to the space $C(\mathbb{C})$, demonstrated in $[3,13]$.

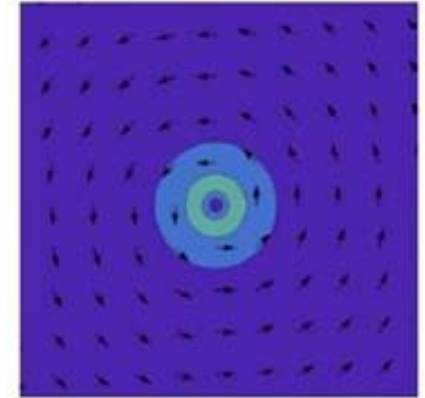

(a) Vector modeling of rotation magnetic ring

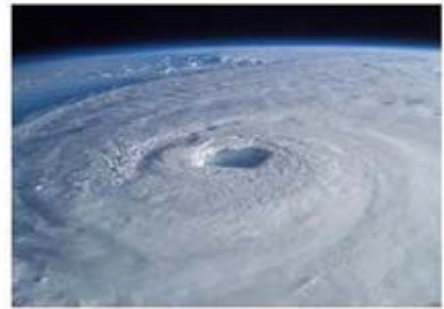

(d) Twisted surface of cyclone

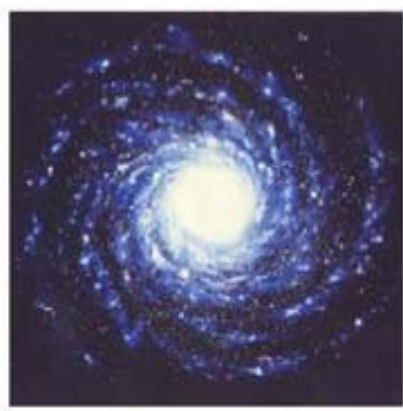

(b) Spiral galaxy

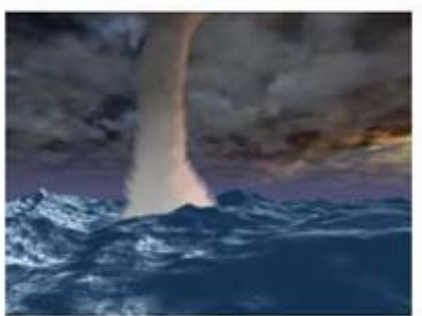

(e) Twisted surface of thunderbolt

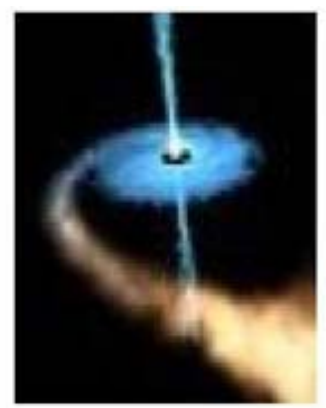

(c) Black hole

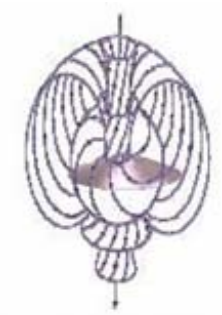

(f) Our synchrotron ship (with an electro-gravitational field)

Figure 3. Rotations and superconducting effects with concentric condensed matter plays an important roll in the movement and performance of the many objects that haves twistor surfaces with high forces of impulse and displacements through of vanishing space of continuum media.

we have the execution of the action of the Maxwell fields $[1,18]$,

$$
\begin{aligned}
& \operatorname{Exe}_{\text {MAX }}: \mathfrak{I}(x(s)) \mapsto \int_{\gamma}\left(\int_{M} L_{\text {MAX }}(x(s)) \mathrm{d}(x(s))\right) \mu_{s} \\
& =\int_{\gamma} L_{\text {MAX }}(x(t), \dot{x}(t)) \mu_{t},
\end{aligned}
$$

where $\mu_{s}$ is the corresponding measure of the trajectory $\gamma$, in $M$. But the system we want to build must be considered the by action $\mathfrak{I}$, of the electromagnetic field $F$, to suspend or make levitation and to displace an object $\mathcal{O} \subset$ $M$, in the space $M$.

The laws of conservation of the electromagnetic fields which establish the levitation and movement of trans- lation of the object $\mathcal{O}$, (vehicle) establishes that the energy of $\mathcal{O}$, under this constant frame comes as

$$
E_{O}=E_{K}+H\left(A, \Im_{M}\right),
$$

where $L_{M A X(S C)}$, is the corresponding Lagrangian of Maxwell of the superconductor SC. Likewise, the action given by $\mathfrak{I}_{M}$, is will transmitter to all particle $x(s)$, of $\mathcal{O}$, characterises it like a superconductor.

If we consider the global evaluation of the operator that acts on the space of points $x(s)$, this comes given for Equation (6), as (this is magnetic conscience operator! $[4,6])$ :

$$
\mathfrak{I}_{M}: x(s) \mapsto \int_{O} L_{M A X(S C)}(x(s)) \mathrm{d}(x(s)) .
$$


But in that global action of the magnetic field on the superconductor, the thermodynamically effects mentioned of the field arisen as meaningless part in the process $[10,16]$, is to say

$$
\mathfrak{I}_{M}(x(s))=L_{M A X(S C)}\left(\Psi_{n}(V, T)\right)+\Omega \int_{O} H^{2} \mathrm{~d} V,
$$

where $\Omega$, depends on the geometrical form of $\mathcal{O}$, in our case the design of the vehicle will be an ellipsoid or a disc, convenient geometries of the form of the vehicle that will be discussed after and that represents intermediate superconductors states in the region of interaction of field [10]. Furthermore, these states establish the specific topological transforms in the superconductor context [10], and by physic of superconductors are related [12].

The term $L_{M A X(S C)}\left(\Psi_{n}(V, T)\right)$ explains the thermodynamics effects of the action of the field in the neighborhood of the reactor. If there isn't these magnetic effects then $\Omega \int_{O} H^{2} \mathrm{~d} V=0$, and $\Im_{M A X(S C)}(x(s))=L_{M A X(S C)}\left(\Psi_{n}(V, T)\right)$ $=F(V, T)_{0}$, is the corresponding thermodynamic action that only depends of the volume (size of the reactor), and not of their form [12]. $\mathrm{T}$ is the temperature and this is an effect obtained for the reactor operation.

In the case of the kinetic energy, this depends of their rotation, which is an effect of the action of their magnetic moment of spin. To our object $\mathcal{O}$, in the case of that is an intermediate state of a superconductor, the produced energy to their electromagnetic impulse provided of the twistor surface that is generated under $\mathcal{O}$, and whose action is given by the action of the corresponding moment of rotation. This twistor surface is a magnetic field whose action is the synergic impulse of the vehicle using the energy of the same like superconductor. Of this form, the total action of auto-levitation and movement on the vehicle $\mathcal{O}$, will come given by the equation

$$
\mathfrak{I}_{O}=\mathfrak{I}_{M}+\mathfrak{I}_{\text {rot }},
$$

whose energy is given by (9), with execution operator given for

$$
\begin{aligned}
& \text { Exe }_{\text {MAX }}: \mathfrak{I}_{M}(x(s)) \mapsto \\
& \int_{\gamma}\left(\int_{O}\left\{L_{\mathfrak{\Im}_{M}}(x(s))+L_{\text {rot }}(x(s))\right\} \mathrm{d}(x(s))\right) \mu_{s} \\
& =\int_{\gamma}\left\{L_{\mathfrak{I}_{M}}+L_{\text {rot }}\right\}(x(s), x(\dot{s})) \mu_{s},
\end{aligned}
$$

and which is transmitted to whole particle $x(s)$, of the structure of $\mathcal{O}$, through the law of conservation of magnetic energy in all moment in the process of the superconductor.

Lemma. 1.1. (Bulnes, F) [8,14]. The energy of action given by $\mathfrak{I}_{\mathrm{M}}$, of the vehicle $\odot$ all like a diamagnetic given by $\mathfrak{I}_{M}$ satisfies all vector magnetic potential $A$, (1-form of the corresponding Maxwell equations to the levitation: $\operatorname{rot} B=4 \pi j(1 / c)$ and $B=0,[10,13])$, the following Hamiltonian

$$
H\left(A, \mathfrak{J}_{M}\right)=\int_{O}\left\{L_{M}-H^{2} / 8 \pi\right\} \mathrm{d} V .
$$

Proof. See [8], and [16].

One can obtain considering the synergic action prosaid in (13), the following synergic principle deduced of our Equations (12), (13) and (14):

Principle: "The force that exercises the imam (it leaves conformed by magnetic rotation ring), and superconductor is the sum of contributions of each element of local surface and each one of them is the double that would correspond to the direct force among the local image (superconductor) and the imam".

This clearly satisfies the property of our integral synergic operators given in (12):

$$
\int_{M} \mathfrak{I}(x(s)) \mu \geq \sum_{j} \int_{O} \mathfrak{I}_{j}(x(s)) \mu_{j},[14,17]
$$

Considering the combined actions, we can give a general scheme to design a possible reactor that, see Figure 4 and Figure 5.

\section{Electromagnetic Flight Overdrive, Patent of Constitutive Components of the Ship Reactor and Their Magnetic Displacement}

Definition. 2.1. A $\mathfrak{E}_{t} \otimes \mathbb{Z}_{\text {G }}$-field, is an element of a bisided ideal of the Maxwell fields $[1,6]$. Explicitly is the formal space:

$$
\begin{gathered}
\mathfrak{E}_{\mathrm{t}} \otimes \underset{\boldsymbol{F}_{1}}{=}=\left\{\left(F_{1}, F_{2}\right) \in \Omega^{2}(O) \times \Omega^{2}(O) \mid F_{1} \otimes F_{2}\right. \\
\left.+F_{2} \otimes F_{1}-\left[F_{1}, F_{2}\right], \text { with } \otimes=\otimes_{\mathbb{R}}\right\},
\end{gathered}
$$

Lemma. 2.1. All electromagnetic actions (microscopic and macroscopic) used to the flight and movement of $\odot$, comes of the $\mathbb{E}_{t} \otimes \mathbb{1}_{-1}$-fields. See Figure 6.

Proof. To demonstrate this result it is necessary prove two aspects that are correlated: The microscopic properties due to the macroscopic effects produced for the reactor to characterize the quantum densities of field to superconductor electrons $e, 2 e[5,15])$, which comes given by the auto-adjunct part:

$$
J_{s}=\frac{e^{*} h_{M}}{2 m * i}\left(\psi^{*} \nabla \psi-\psi \nabla \psi *\right)-\frac{\left(e^{*}\right)^{2}}{m^{*} c}|\psi|^{2} A, \quad[8,12,17],
$$

and the macroscopic effects due to the Eddy currents which must comply the following equation of electromagnetic field $[8,10]$ :

$$
F=\left(H_{i} H_{k}-\frac{1}{2} H^{2} \delta_{i k}\right) / 4 \pi \sigma \mu,
$$

Consider the bi-sided ideal given by the space (16), whose actions are extended to all space from $\mathcal{O}$, until the infinite (ambient of $\odot$ ) $[7,10,19]$. By the Lemma 1 ( $F$. 


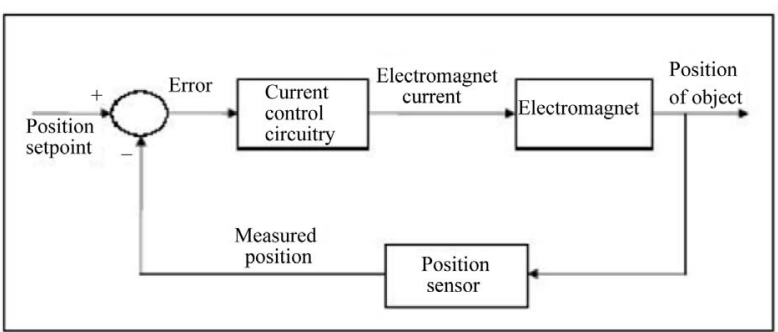

Figure 4. General diagram of control of a levitation system with synergic action on set points of an object. The synergic action comes given for the (13), and under the law conservation of the energy established for the (9).

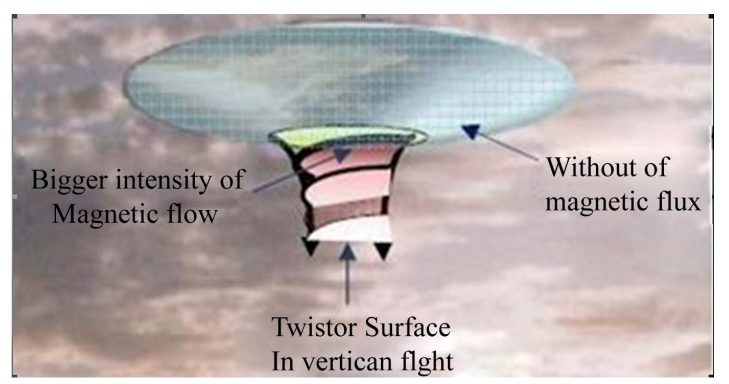

Figure 5. Rotation ring conforming the twistor surface into magnetic context. Expulsion of the magnetic field by the superconductor on the surface of the vehicle (part in blue of the plate). Both actions in a synergic, like established in (14), due to the superconductor and ring actions must be synchronized to the adequate behavior of vehicle in full flight. Thus, we can name it to effects of our technological patent like electromagnetic synchrotron vehicle.

Hyperbolic waves induced by eq.17,

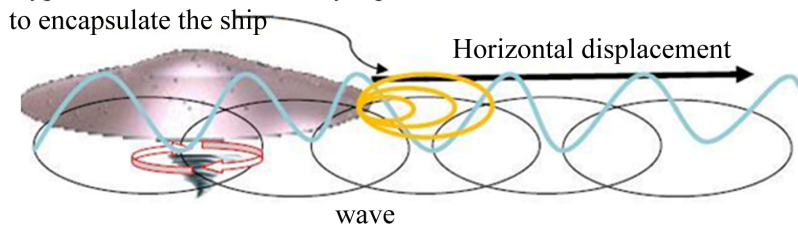

Figure 6. The figure shows how it could displace it horizontally using the magnetic energy foreseen by the equation (18). In this same tenor the ship through an emission of magnetic waves of hyperbolic type (deduced of the hyperbolic structure of (18)) that established a magnetic shield that cut the media producing a displacement like magnetohydrodynamic wave very similar to the submarines. The ship stays encapsulated by the magnetic wave this begins their horizontal movement [10].

Bulnes), page 5, (published in ASME, 2010), the quantum effects underlying in superconducting phenomena satisfies that

$$
\begin{aligned}
& H\left(A, \mathfrak{I}_{M}\right)=\mathfrak{I}_{M}-H(A, B)=\int_{O}\left[F_{i} F_{k}-\frac{1}{2} H^{2} \delta_{i k}\right] \mathrm{d} V / 4 \pi \\
& =\int_{O} L_{M}(x(s)) \mathrm{d}(x(s))-\int_{O} H^{2} / 8 \pi \mathrm{d} V,
\end{aligned}
$$

where $H^{2} / 8 \pi$, is the free magnetic energy $\left(B=H / 4 \pi \mu_{0}\right)$, and the integral of the Lagrangian $L_{\mathrm{M}}$, of the expulsion by the action $\mathfrak{I}_{\mathrm{M}}$, and that is useful to establish the macroscopic wave functions that give place to a microscopic quantum current $J_{\mathrm{s}}$. In effect, by mathematical electrodynamics [4], we can define using the structure of $\mathbb{E}_{\mathrm{t}} \otimes \mathbb{1}$, (that is to say, a module of the exterior algebra (which is deduced by the universal map applied to each term of the element $\left.\left.F_{1} \otimes F_{2}-F_{1} \otimes F_{2}-\left[F_{1}, F_{2}\right]\right)[1,7]\right)$ that:

$$
(\psi, J)=\int_{\partial O}\left(\psi^{*} \wedge J-J \wedge \psi^{*}\right) \mathrm{d} S-\int_{\partial O}\left\langle\psi, \psi^{*}\right\rangle \mathrm{d} S,
$$

where $J=\nabla \psi$, and $\psi^{*}=\operatorname{conj}(\psi)$, such that $|\psi|^{2}=$ $\left\langle\psi, \psi^{*}\right\rangle$. That is what we want, since we search measure energy. A study on isometric and complete Riemannian manifolds [16], can clarify more the existence of the Hermitian product deduced into the energy definition by tensors $F$, and conserve the invariance of this product $[1,16]$. Like the last integral, (19), measure effects due to the macroscopic actions to quantum level, this proves the first affirmation. The second affirmation (17), is deduced by a volume integral like the given for (18). Thus the total current $J_{\mathrm{s}}$, is the extend on all surface of vehicle $\mathcal{O}$, such and as it is have foreseen to characterize to $\mathcal{O}$, like diamagnetic material in the space. The microscopic effects succeed due to the electromagnetic actions and under the conservative laws foreseen by our integral (13), [8], since that process to obtain condensed matter to microscopic levels, producing the macroscopic levels observed in the sidereal objects $[6,9]$.

In fact, the integral given by (19), can be calculated for the stokes theorem $[1,8]$, where $j=\operatorname{rot} B$, on surface $S$, of the superconductor, having a total magnetic flux on all surface of $\mathcal{O}$.

How to characterize and produce artificial materials that induce the same microscopic and macroscopic effects necessary into the magnetic conscience induced by the deep conscience (programmed by the structure space defined by (16)), to obtain a superconductor state in the reactor to ambient conditions?

An important thermal fact is observed in the study of superconductors. If $T_{\mathrm{c}}$, is the critical temperature of a material to risk transition to their superconductor characterization (foreseen by (11)), then is clear that if $T$, is a temperature of any material in nature condition to be conductor, and if we want characterize it like superconductor is necessary that $T_{\mathrm{c}}>T$, [12]. See Figure 7. The same material to reciprocate inequality not exits like superconductor.

For other side, magnetic effects are intimately related with the microscopic effects that are produced by the quantum macroscopic effects where these last are gene- 


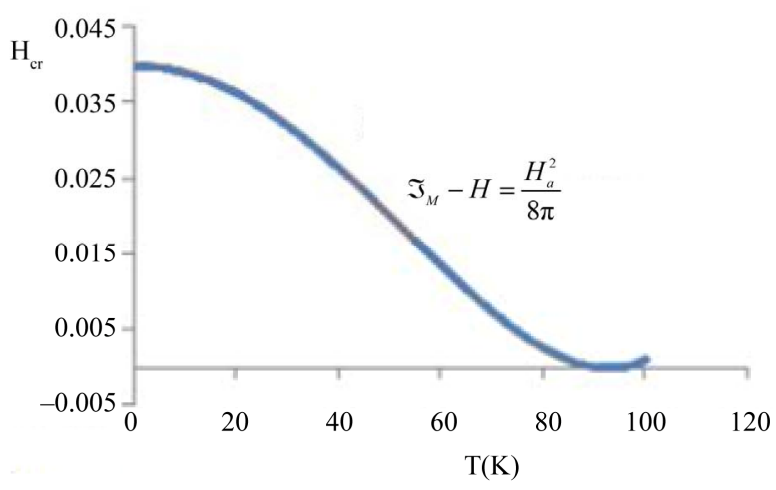

Figure 7. Shows the curve of critical magnetic field versus temperature depending of volume [8,13].

rated for the magnetic field at all moment will produce the diamagnetic effect on the ship, such and like is predicted in the (11). But into superconductor theory the element ambient also has inherence into the action $\mathfrak{I}_{\mathrm{M}}$, (of fact this also will be relevant in the displacement of the ship like a wave and will produce condensation effects such and like has been signed into the demonstration of the Lemma. 2. 1 see the Figures 6 and 8 ). Thus the material that we must think must be a material with the sufficient qualities like conductor with propitious properties to diamagnetism (that is to say with components of conductor metals with a magnetic susceptibility adequate to risk the transition state), and qualities of not rapidly decreasing in a short interval to reliever of oxygen atoms in a special alloy thought-out to the construction of the reactor. Precisely the existence of oxygen atoms can give us the condensed matter effects from quantum level, that here in the atmospheric continuum media, will do that the flight be relevant with high qualities like traveler magneto-hydrodynamic wave, including mimetic or camouflage effects. The clouds are condensed water in the sky!

We suggest the following patent of alloys rich in oxygen atoms, with a functional relation to the number of oxygen atoms related with tetragonal configuration with oxygen of the $\mathrm{Cu}$-metal, and proportional parts of metals such how the Ba (Barium), Y (Yttrium), La (Lanthanum), inclusive $\mathrm{Yb}$ (Ytterbium). But always Cuprum present [12].

Proposition. 2.1. The inequality $T_{c}>T$, risks a larger interval of the molecular bi-atomicity parameter to Oxygen atoms, when the alloy is of the family of the type, YBACUO $(\zeta)[12,15]$ (because it is require maxim $T_{c}$ ) that which it is get when in the functional space $\sim \mathrm{II}_{e}(j, \rho)$, [6], (fluid magnetic space that is superconductor with charge density $\rho$, and $j \in \mathfrak{I}_{\rho} \oplus \mathfrak{I}_{0}$, is the total induced current by the magnetic field $H$, in the ship), comply in their superconducting characteristic (patent of component in process $P M x[20])$ that:

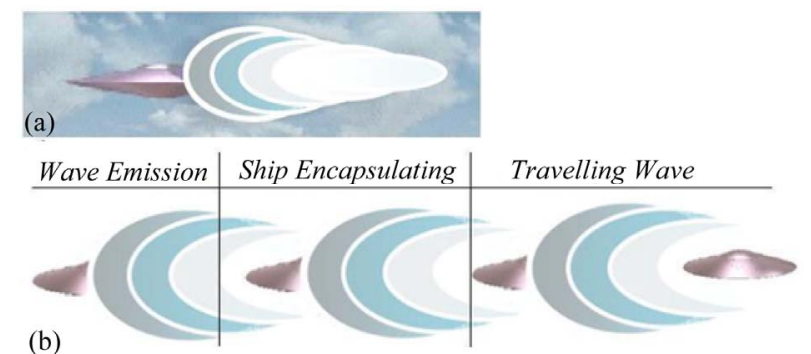

Figure 8. (a) Describes the emission of electromagnetic waves that will be useful to horizontal and change of spatial direction of the ship; (b) Describes the displacement of the ship like travelling wave.

$$
\begin{gathered}
\sim \mathrm{II}_{e}(j, \rho)=\left\{j \in \mathfrak{I} \rho \oplus \mathfrak{I}_{0} \mid j=\left(\rho^{-} \mu^{-}+\rho^{+} \mu^{+}\right)\left\{j_{0}+j_{\mathrm{e}}\right\},\right. \text { such } \\
\text { that rot } B=4 \pi j(1 / c)\},
\end{gathered}
$$

with the Eddy currents $[8,10]$,

$$
\Delta \boldsymbol{H}=\frac{4 \pi \sigma \mu}{c^{2}} \frac{\partial \boldsymbol{H}}{\partial t},
$$

Proof. See [10], and use an analogy of the consequences of the Helmholtz theorem on material drafting and superconducting conditions under inner-sidereal magnetic field [6,9], (remember that our prototypes of ships are similar to sidereal objects as galaxies or stars).

In the disc obtained by the rotation and superconducting transformation [21], it is have two orbital spaces $P^{+}$, and $P^{-}[1-3,6]$, that are consequences of the expulsion of the magnetic field by the superconductor of the reactor. In these orbital spaces generates the currents $\rho^{-} \mu^{-}\left\{j_{0}+\right.$ $\left.j_{e}\right\}$, and $\rho^{+} \mu^{+}\left\{j_{0}+j_{e}\right\}$, where the sign $+/-$, indicates the direction (oriented orbital spaces) of the current lines of the current density. The magnetic field degenerate in infinite magnetic line with residues to quantum level, such and as comes pre-whose in the (18). The parameter $\zeta$, must be designed to give a material that under conditions of temperature given by $T_{\mathrm{c}}>T$, realizes the actions established in (9), but this is obtained with the family $I B A C U O$, that which among other things (and using the diamagnetic effect due to the Eddy currents) produces an effect of diamagnetic field, comparable with the Earth magnetic field; (namely, $\approx 0.1$ gauss $[11,12]$ ), producing the suspension and levitation effects necessary to levitation and suspension to ambient conditions of temperature. But this situation is only satisfied into a space with similar conditions like the given in the sidereal space, that is to say, the space $\sim \mathrm{II}_{e}(j, v)$.

\section{Microcontroller of the Specific Eddy Flux and Iso-Rotations in the Superconductor Reactor}

Using all the before study foreseen on Eddy currents and relating the behavior of magnetic flux with skin effect 
given to the Eddy currents on surface of vehicle $O$ (considering the macroscopic quantum effects), and studied in $[10,12]$, and [16], we can enunciate the following result to obtain a sensor chip to control the pressure of the Eddy currents for unit of surface of $O$ :

Theorem (F. Bulnes) 3.1. We consider (18), and $F_{s}=-H_{e x t}^{2} \boldsymbol{n} / 8 \pi \sigma \mu$ with the Hamiltonian foreseen in (14), by lemma. 1., and their proof, it is have of inequality on magnetic energy necessary to all magnetic process of reactor

$$
8 \pi \Phi_{0} n H_{v} \geq \int_{o}\left(H\left(1-h \nabla^{2} \boldsymbol{H}\right)\right)^{2} \mathrm{~d} V \geq 8 \pi \int_{o} H^{2} \mathrm{~d} V
$$

Then a sensor-chip to magnetic flux (pressure on the surface of $O$ ) to super-currents is defined by the inequality

$$
\int_{V} j_{s}(x, y) \delta(z) \mathrm{d} V \leq \int_{S} B \mathrm{~d} S \leq n \Phi_{0},
$$

where $\Phi_{0}$, is a fluxoid $\left(=h \mathrm{c} / e^{*}=2.07 \times 10^{-7}\right.$ gauss $\left.\cdot \mathrm{cm}^{2}\right)$, being $e^{*}=2 e$, where $\mathrm{e}$ is the charge of electron.

Proof. See [8], and consider a superconductor of type I, taking the intermediate state [10], to calculate the transition to type II, with $\lambda(T)>\xi(T)$. Then apply the Stokes theorem to calculate the super-currents through magnetic field by fluxoids in each component of the super-canonical algebra $\mathfrak{E}_{\mathfrak{t}} \otimes \mathfrak{I}_{\mathrm{f}}$, to quantum level $[7,8,12]$. Of this manage; we obtain the magnetic energy required for our levitation and suspension of vehicle.

\section{Simulations and Prototypes}

Let the magnetic energy foreseen in the Lemma. 1.1, together with the necessary geometric considerations to engender the twistor surfaces in the area of fluxoids existence (Lemma 2.1), then we can obtain by means of a specialized simulation program in magnetism [22], the distribution of the magnetic flow $\Phi$, see Figures $\mathbf{9}$ and $\mathbf{1 0}$ under and of profile of a geometric model designed with foundation in the Lemma 3, (one model of built disk under a special alloy).

After obtaining a micro-controller of Eddy Currents deduced of the interaction of the superconductor with the magnetic flow already obtained by the rotation ring with certain magnetic moment predicted (Lemma 2), it is also necessary to program the micro-controller so that it stabilizes the object (our vehicle), [23], when it is located in a combination of variable magnetic fields. This is achieved because when the object is attracted in an address anyone for effect of one of them, the system weakens the corresponding field to avoid or to diminish to the maximum this movement, as long as if goes away in opposed sense, the system realize the contrary action. Only is it required that the answer of the system is the sufficiently quick

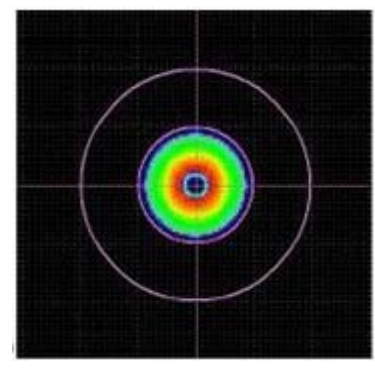

(a)

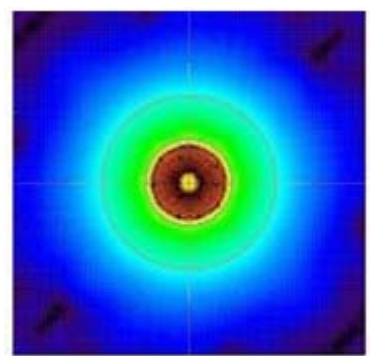

(b)

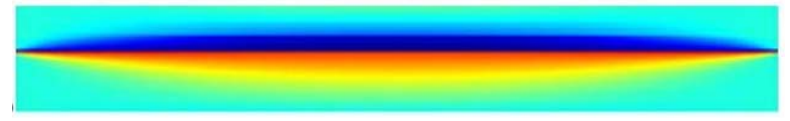

(c)

Figure 9. The incise (a), shows the magnetic flux that emanate of the superconductor when this is not avoided. The colored areas in red denote bigger intensity of magnetic flow. Let us observe that these appear in the ring of rotation of the vehicle and very near to the limit with the cavity or inferior opening of the vehicle that is in the central inferior part to the vehicle. In the incise (b), the center is a cavity for where the twistor will come out engendered by the lines of rotation of the magnetic field of the ring. The part colored in blue determines the absence of magnetic flow, such like it is wants that it happens for the super-currents existence on the surface of the vehicle, making that this everything behaves as a diamagnetic, except in the mentioned ring region. In the incise (c), one has a view of profile of the magnetic flow of our flying plate (This simulation was published in the Proceedings of Fluid Flow, Heat Transfer and Thermal Systems of ASME in the paper IMECE2010-37107, British Columbia, Canada, with all rights reserved $\left.{ }^{\circledR}[13]\right)$.
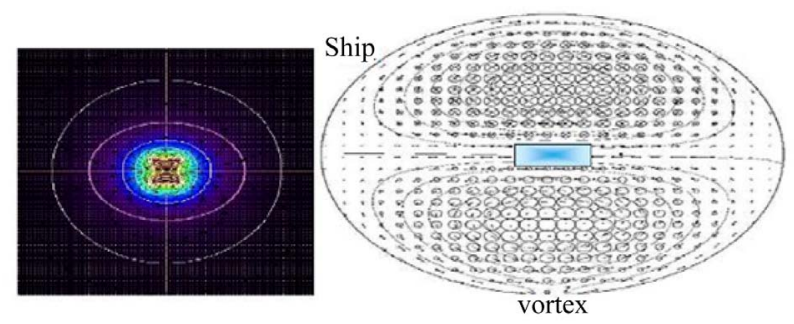

Figure 10. External magnetic field to superconductor reactor in a vehicle Prototype I. The reactor of the ship is the blue rectangle [22] and super-conductor-magnetic spectral simulation of Prototype I.

thing so that the object does not oscillate or that, in any event, its movement is minimum. To achieve the objective of manipulating the magnetic fields we have to design our own computation architecture that establishes and optimizes the use of memory and the time of processor to manage in parallel all the variables to process. This prosecution system is designed with FPGA (Field Programmable Gate Array) for implementation of the system prosecution) in such a way that we control the variables [22], involved as the curvature of $E(k)$ and the 
dispersion relationship $E(K)$ being necessary in this system to control the vibrations of the atoms of the net in the solid.

The rotation of the ring (iso-rotation in a plane), it is managed in the variable of the vector $z(k)$ to feed the mechanical system $[15,22,24]$. The magnetic field is controlled by means of the acquisition by means of the adjustment of the probes of the sensors that feedback to the system. See Figure 11.

\section{Conclusions, Future Developments and Measurements in These Directions}

In this moment we are in the preparation of the real prototypes with all the mechanism and devices that will do possible and feasible the flight of our design and development of the vehicle. See Figure 12. In the soon months we will realize the first electronic rehearsals and real proofs of the prototypes using the concrete facts and other observations which aren't included in this research paper for reasons of still being unfinished. A problem that it is have is the fabrication of the material to the reactor with a pure percent adequate win the gravitational effects to quantum level in the process of obtaining the twistor surface of levitation and suspension of the ship. In some cases the design of the prototype I, results more convenient to the porpoises of levitation to low velocities and to conform the direction of the horizontal or directional displacements since the protuberance of the extreme the ellipsoids are propitious to the emission and transmission of waves to encapsulate process to flight like a wave (obviously derived of their geometry). The corresponding micro-controller of the reactor must control the flux of the Eddy currents through the signals which is encoded by the electromagnetic action defined by the (6), and that providence of a permanent electromagnetic field the which it is self-generated for the interactions given by superconductor and magnetic field. In fact, the iso-rotations cited in the lemma given in [8], are also generated by the same permanent field. The device to control the dosage of the Eddy currents flux will give the intensification of the property of diamagnetism to the vehicle and with it the magnification or minimalism of the actions during of the flight. Finally, the generation of an electro-gravitational field or $\mathfrak{E}_{\mathrm{t}} \otimes$ 暞 -field (diamagnetic field that must vender the gravitational forces) for permanent waves with velocities of major phases that the light velocity can create a macro-quantum with the superconducting effects of the fluxoids calculated and foreseen by the Inequality (23), and that will also produce the Eddy currents in the encapsulate of the ship like if it were an enormous particle with the foreseen geometrical form (a plate, a sphere or an ellipsoid), can be associated like

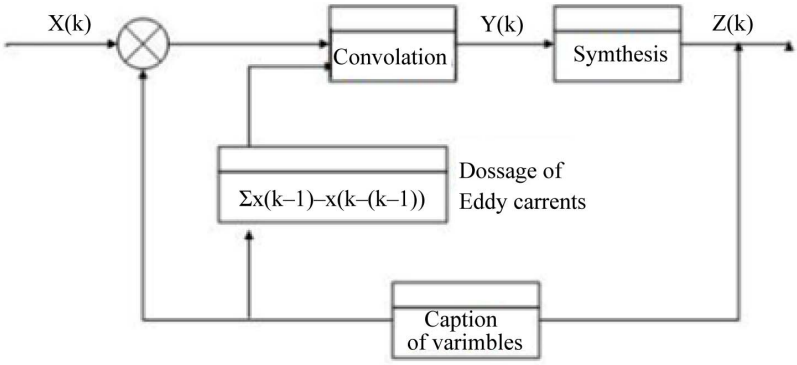

Figure 11. Diagram of the control system $X(k)$, is the vector of the shown variables. $Y(k)$, it is the feedback signal and $\mathrm{Z}(\mathbf{k})$, is the signal of control answer. In the central block after the reception of variables, Eddy's currents are dosed. (This control design was published in the Proceedings of Fluid Flow, Heat Transfer and Thermal Systems of ASME in the paper IMECE2010-37107, British Columbia, Canada, with all rights reserved $\left.{ }^{\circledR}[13]\right)$.

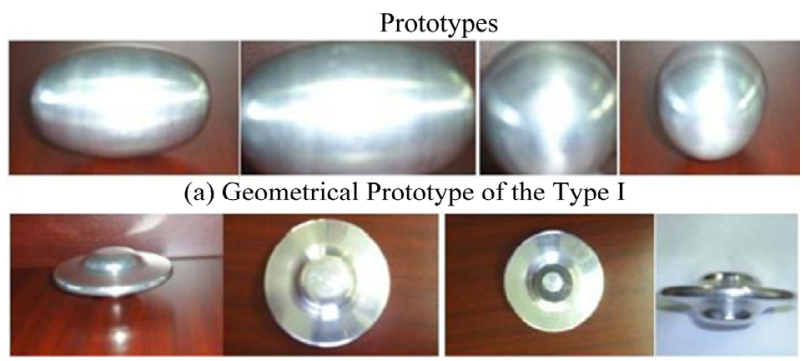

(b) Second Geometrical Prototype of the Type II

Figure 12. The considered prototypes are based in the geometrical considerations given by Lemma. 2.1, (published in [13]), and electromagnetic interactions between superconductor and magnetic field auto-generated by these interactions, by Lemma. 3.1, and theorem. 4.1. The two prototypes must be of special materials in order to obtain the effect of superconductivity to normal ambient conditions. The material must be of alloy $\mathrm{Y} / \mathrm{E}-\mathrm{H} / \mathrm{Mx}$ is obtaining the nano-effect by fluxoids in structure predict by Lemma. 2.1, and theorem. 4.1. (This prototype designs were published in the Proceeding of Fluid Flow, Heat Transfer and Thermal Systems of ASME in the paper IMECE2010-37107, British Columbia, Canada, with all rights reserved $\left.{ }^{\circledR}[13]\right)$.

quantum macro-particles using the Bohm re-interpretation $[1,2]$, in an analogous model to quantum scheme to the macroscopic scales mentioned with before (19), that using the equivalences defined by the isomorphism of the cohomological classes $\mathrm{H}^{1}(\Pi(X), \Omega) \cong \mathrm{H}^{1}(\mathrm{P} \mathcal{T}, \mathrm{O}(-2-2))$, (see the meaning of the notation used to characterize the spaces mentioned in this work), and with images in the cohomological class $\mathrm{H}^{2}(\mathcal{O}, \mathbb{R})$, (integrals that are functional), the wave functions can be re-interpreted like vertexes of a graph of displacement trajectories to consider the translation of the vehicle, being able to design a ship control (based in the design of the Figure 4), like a macro-particle (the vehicle and their electromagnetic 
revetment), in the space. Given that the particles conforms the macro-particle that define the vehicle in this transitory process of displacement like traveler wave, it has a proper auto-energy of their atoms, thus the vehicle will have a quasi-unlimited auto-energy being achieved by it for example, the instantaneous movement to a spatial position. This will give the more efficient form of displacement the vehicle. The wave function related to the vehicle could be changed causing a field around of the vehicle doing it asymmetric (such as it pre-says the space defined in (16), and their density of energy given in (18)). This is equivalent to cause a change of phase related with the macro-particle condition of the vehicle. This action could be realized easily and instantaneously. The vehicle could realize a "jump" defined by displacement contemplated by the Figure 7, to new spatial position (some seemed to Warp flight $[4,6]$, but different as for their interaction with the media, and under an action of the reactor on the vehicle producing the quantum macroscopic effects foreseen like wave in (18). In the transitory process of displacement and for the quantum effects produced for the reactor and the magnetic field of the vehicle, this is diffracted producing an effect of invisibility apparent. Of a more precise manage we can affirm that the effect is produce due to that the superconductor material is an alloy whose nano-crystal structure has the properties of that curve the light, at least for the presence of some of the atoms of the metals that it composes, producing an effect of curvature in the light, the which generate an apparent receiving of the vehicle, and temporally invisible (similar to the eclipses, although by distinct reasons to the gravity).

The future designs of ships must be in functionality and behavior very seemed to the sidereal and natural objects that exist in the universe, since in these natural objects fulfilled the unlimited energy to use the proper object to displacement and other functions that designed these. In this moment are about to obtain the exact alloy with high qualities to obtain all the effects described in this work. The problem that we have is to obtain the enough financing to do it. We hope to obtain it soon.

\section{REFERENCES}

[1] F. Bulnes, "Foundations on Possible Technological Applications of the Mathematical Electrodynamics," Masterful Conference in Section of Postgraduate Studies and Research (SEPI), National Polytechnic Institute, Mexico, 2007.

[2] F. Bulnes, "Doctoral Course of Mathematical Electrodynamics," Proceedings of the 2nd Appliedmath, Vol. 9, 2007, pp. 398-447.

[3] F. Bulnes and M. Shapiro, "On General Theory of Integral Operators to Analysis and Geometry (Monograph in
Mathematics)," IM/UNAM, Mexico, 2007.

[4] F. Bulnes, "Treatise of Advanced Mathematics: System and Signals Analysis," Faculty of Sciences, UNAM, Mexico, 1998.

[5] F. Bulnes, "The Super Canonical Algebra $\mathrm{E}_{\mathrm{t}} \otimes \mathrm{H}$," International Conferences of Electrodynamics in Veracruz, IM/UNAM, Mexico, 1998.

[6] S. Nagaya, K. Komura, N. Kashima, M. Minami, H. Kawashima, Y. Nara and H. Ishigaki, "Influences of Separate Position to Radial Direction between Bulk Superconductor and Permanent Magnetic Ring about Magnetic Levitation and Rotating Characteristics," Physica C: Superconductivity, Vol. 392, 2003, pp. 754-758. doi:10.1016/S0921-4534(03)01011-6

[7] J. A. Díaz, "Systematization of the Design of Devices of Superconducting Levitation by Meissner Effect," Ph.D. Thesis, Institute of Mechanical Engineering, University Carlos III of Madrid, Spain, 2008.

[8] M. A. Alario and J. L. Vicent, "Superconductivity," Complutense University, Madrid, 1991, pp. 170-234.

[9] F. Bulnes, "Conferences of Lie Groups. SEPI-IPN and IM/UNAM," Section of Postgraduate Studies and Research/IPN, Mexico, 2005.

[10] F. Bulnes, "Special Dissertations of Maxwell Equations," Only registered SEP, Mexico, 1996, unpublished.

[11] D. Werner, "Einführung in Die Theoretische," W. de Gruyter \& Co., Berlin, 1963.

[12] I. M. Gel'fand, I. M. Shapiro and I. Graev, "Generalized Functions," 2nd Edition, Academic Press, New York, 1965.

[13] F. Bulnes, E. Hernández and J. Maya, "Design and Development of an Impeller Synergic System of Electromagnetic Type for Levitation, Suspension and Movement of Symmetrical Bodies," Proceedings of the Fluid Flow, Heat transfer and Thermal Systems of International Mechanical Engineering Conferences and Exposition/ ASME, British Columbia, 12-18 November 2010.

[14] L. D. Landau and E. M. Lifshitz, "Electrodynamics of Continuous Media (Volume 8)," 2nd Edition, Pergamon Press, London, 1960.

[15] M. Peimbert, "Selected Themes of Astrophysics (Compilation by Peimbert)," Faculty of Sciences, UNAM, Mexico, 1984.

[16] B. Simon and M. Reed, "Mathematical Methods for Physics, Vol. I (Functional Analysis)," Academic Press, New York, 1972.

[17] M. Okano, N. Tamada, S. Fuchino, I. Ishii and T. Iwmoto, "Numerical Analysis of a Superconducting Bearing," IEEE Transactions on Applied Superconductivity, Vol. 10, No. 1, 2000, pp. 909-912. doi:10.1109/77.828379

[18] K. B. Postrekhin and W. K. Chu, "Superconductor and Magnet Levitation Devices," Review of Scientific Instruments, Vol. 74, No. 12, 2003, pp. 4989-5017.

[19] J. E. Marsden and R. Abraham, "Manifolds, Tensor Analysis and Applications," 2nd Edition, Addison Wesley, 
Massachusetts, 1982.

[20] F. Bulnes, "Analysis of Prospective and Development of Effective Technologies through Integral Synergic Operators of the Mechanics," Proceedings of the 5th Cuban Congress of Mechanical Engineering, Havana, 2-5 December 2008.

[21] N. N. Fiodorov, "Foundations of Electrodynamics," 3rd Edition, Mir Moscow, Moscow, 1983.

[22] Program of Magnetism, Vizimagtr318.

\section{Technical Specialized Notation}

$\omega_{H}$-Rotational Magnetic Field.

$L_{\mathrm{MAX}}$ - Lagrangian Operator of Maxwell.

$j_{\mathrm{s}}$-Superficial currents (are the super-currents of Eddy currents).

$L_{M}$-Lagrangian Operator of Maxwell it comes of the magnetic action.

$\mathfrak{I}_{M}-$ Action of magnetic field.

$F$-Tensor of Maxwell or electromagnetic tensor (is a fundamental 2-form defined by $F=F_{\mathrm{ij}} \mathrm{d} x_{\mathrm{i}} \mathrm{d} x_{\mathrm{j}}$ ).

$J_{0}$ - Functional space of currents proveniences of magnetic field with the condition $\operatorname{rot} H=j$.

O-Vehicle or flight ship under principle of magnetic impulse, levitation and/or suspension.

$v$-Velocities field.

$O$ - Integration domain of the vehicle $\mathcal{O}$.

$\widehat{O}_{\mathrm{M}}$-Reactor of magnetic impulse, levitation and/or suspension of the ship or flight vehicle $\mathcal{O}$ (is composite for the superconductor and magnetic ring).

$J_{\rho}$-Functional space of the electrical currents proveniences from electric field.

$C(\mathbb{U})$ - Space of classes that are submanifolds of a maximal complex torus. Such submanifolds are toroids or rings. To our study case, these are of magnetic origin and are produced by rotation and the superconductor action of $\mathcal{O}$.

G/C $(\mathbb{C})$ - Homogeneous space of classes that admit Riemannian complex invariants structures, that means, invariants complex surfaces under the action of the compact group $K$. To our case, the group of the electromagnetic field action operators, which is $K=S U(2)$.

$S U(2)$ - Topological group of the electromagnetic fields action operator $S U(2)=\left\{F \in \Omega^{2}\left(\mathbb{R}^{4}\right) \mid F_{\mathrm{ij}}=-F_{\mathrm{ji}}\right\}$. Their elements have linear representations as well matrices of range $2 \times 2$ on $\mathbb{R}$.

A-Vector Potential.

$\Phi$-Magnetic flux. http://www.vizimag.com/

[23] C. Kellum, "Methods and Systems for Generating a Gravity between to Counter-Rotating Magnetic Sources," ACC-theory US20110057754, 2011.

[24] L. P. Hughston and W. T. Shaw, "Twistors in Mathematics and Physics," Cambridge University, Cambridge, 1990, pp. 367-382.
Flux-Flux operator on vector field.

$M-$ Vector of Magnetization.

$\mathrm{H}$-Intensity of magnetic field (In the intermediate state theory, represents their magnetic state).

$\sim \|_{e}(j, \rho)$-Domain Space of an electronic fluid continuum media with dynamical characteristics. Also, from a point of mathematical view is the functional space of total currents from the Magnetic field acting. In electrodynamics is the space conformed for movements caused by the magnetic forces where movements will be observed like the topological distortions of the fluid in the space (twistor surfaces).

$\sim \|_{H}(E, \sigma)$-Domain Space of a magnetic fluid continuum media with dynamical characteristic. Also, from a point of mathematical view is the functional space of the magnetic fields responsible of the transportation of the materials in the ring of the galaxies.

$B$-Density of magnetic flux. Also, vector of magnetic induction.

M-Magnetic moment turn.

$\mathbb{R}^{3}$ - Real space of three dimensions.

$\Omega^{2}\left(\mathbb{R}^{4}\right)$ - Space of real 2-forms on a space of dimension four.

$j$-Density of electrical current. Also, vector of density of electrical current.

$L_{M A X(S C)}$-Lagrangian operator of Maxwell of superconductor.

$S C$ - Superconductor.

$n_{\mathrm{s}}$-Normal vector to the surface $\mathrm{S}$.

$\mathrm{H}_{c r}$ - Intensity of critical magnetic field (also it is known as critical magnetic state).

$\mathfrak{I}_{\text {rot }}$-Rotational action (mechanical rotations on the ring of $\mathcal{O}$ due at magnetic moment of turn).

C-Space of the complex numbers of one dimension.

$\mathrm{P}^{+}$- Orbit given by a magnetic torus under of $\odot$ (surface of torus or ring).

$P^{-}$- Orbit given by a magnetic torus over of $\odot$ (surface of torus or ring). 
H(Flux, Potential)_Hamiltonian operator.

$H^{1}(A, B)$ - Cohomological Space of dimension one from $A$ to $B$. This space establishes a duality of objects geometrical and integral precedence (like resulted of the integration process) that has some objects such as forms, functions vector fields, hyper-surfaces, densities, etc. Usually it can be understood like a space of integrals that are functions or other objects as forms or densities.

$H^{2}(A, B)$ - Cohomological Space of dimension two from $A$ to $B$. This space establishes a duality of objects geometrical and of functional precedence (like resulted of the integration process in the evaluation) that has some objects such as forms, functions, vector fields, hypersurfaces, densities, etc. Usually it can be understood like a space of integrals that are functional.

$\mathcal{T}$-Twistor object. In this work the twistor will surfaces magnetic impulse of ship.

$\mathrm{P} \mathcal{T}$-Orbital twistor space. $\mathrm{P} \mathcal{T}=P^{+} \cup P^{-}$.

$\Pi(X)$ - Space designed by densities like singular states or nodes into transition graph of displacement trajectories of the vehicle. Also $\Pi(X)$, is the set of energy states of the ship like macro-particle.

$\mathbb{R}-1$-dimensional space of real numbers.

$\Phi_{0}$-Fluxoid (elemental unit of magnetic vortex).

$M$-Set space of the points $x(s)$, on such act the inter- actions of superconductor and the magnetic field to produce an autonomous levitation effect in $\mathcal{O}$ (space in where is immerse 0 ).

$S O(2)$ - Topological group of the orthogonal rotations in the space $M$. Is a space of range two matrices on $\mathbb{R}$.

$V$-Volume of a superconductor element.

$T_{\mathrm{c}}$ - Temperature of a superconductor element.

$\mathbb{E}_{t} \otimes \mathfrak{I}$ - Super-canonical algebra with structure of Lie algebra to $S U(2)$ gifted with the tensor product $\otimes$, used to explain the quantum effects of macroscopic level and the quantum structure of the densities of field $\psi$, in the quantum equation of the super-current in their auto-adjunct part:

$$
J_{s}=\frac{e^{*} h_{M}}{2 m^{*} i}(\psi * \nabla \psi-\psi \nabla \psi *)-\frac{\left(e^{*}\right)^{2}}{m^{*} c}|\psi|^{2} A,
$$

of fact $\mathrm{E}_{\mathrm{t}} \otimes \mathrm{H}=\left\{F_{12} \mid F_{12}=F_{1} \otimes F_{2}\right\}$ and $J_{\mathrm{s}}$ it inherits the structure of $\mathbb{E}_{\mathrm{t}} \otimes$ 政.

$\psi$-Function of density or density of field.

$P M x$-Mexican design patent.

$h$-Max Planck constant $\approx 6.6256 \times 10^{-34}$ Joules $\times$ seg.

$L_{\mathrm{M}}$-Langragian operator due at total magnetic field $H$.

$L_{\text {rot-Lagrangian operator due at rotational movement }}$ (iso-rotations) that establishes constant rotations in all point of the ring of $\mathcal{O}$ due to their magnetic movement. 